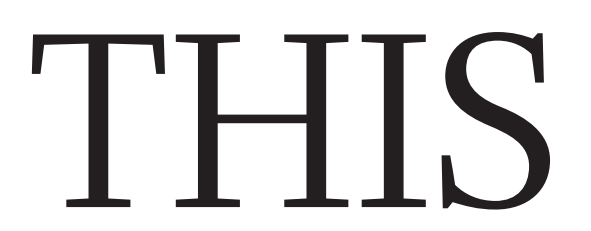

EDITORIALS
HERITAGE Crumbling ruins will make history of Italian history $\mathbf{p . 4 0 0}$
WORLD VIEW WHO offers a new vision for global fight against tuberculosis $\mathbf{p . 4 0 1}$
SQUID Marine luminescence study reveals nervous flashes $\mathbf{p . 4 0 2}$

\title{
Be here now
}

\section{New views of quantum theory that can be tested and have practical applications bring welcome echoes of physics past.}

$\mathrm{T}$ The phrase "Shut up and calculate" is popularly associated with the Cornell University physicist David Mermin, who coined it to describe how many physicists use the mathematics of quantum theory without thinking too hard about its deeper implications.

Mermin himself is clearly not content to shut up and calculate. He has mused on the meanings of quantum theory and of physical theory more generally for many decades, notably in his Reference Frame column for the magazine Physics Today. Now, on page 421 of this issue, he considers a new view of quantum theory, called quantum Bayesianism or QBism, and what it implies for a long-standing question. Namely, how can we reconcile our perception that the present moment is special with the relativistic view that space-time is a continuum that reaches from past to future, with nothing to privilege 'the Now'?

Some will see this as a metaphysical question. Certainly, while the gulf remains between the formal machinery of quantum mechanics, with its wavefunctions and probabilities, and our conscious experience of the world, it is hard to see how the question can be framed with the rigour that science usually demands.

Yet QBism offers a way to put us in the picture, even in the absence of a theoretical link between the abstract microworld and the subjective macroworld. It suggests that quantum theory is telling us what an individual can know about a system in the light of what he or she already knows and expects, just as in standard Bayesian probability.

The idea has been given something of a rough ride by physicists, who seem uncomfortable with QBism because they see it as a solipsistic view of the world. Perhaps Mermin's advocacy will secure it a more sympathetic hearing. At any rate, it has the virtue of refusing to ignore quantum theory's long-standing tussle with the role of the observer.

Aside from the merits of the idea, it is striking that Mermin should discuss it at all. Any view of quantum (or indeed classical) physics that borders on the metaphysical has long been out of fashion. Yet the early architects of quantum theory, such as Niels Bohr, Werner Heisenberg and Max Born, had no reservations about examining the philosophical issues it raised, and the problem of 'the Now' troubled Einstein.

Most famously, Bohr and Einstein argued about whether quantum mechanics allowed any room for the idea of realism - of an objective world that exists independently from our efforts to observe and measure it. Bohr insisted that physics was concerned with what we can know, and was silent on the matter of 'how things really are'. He, Born and Heisenberg made claims about quantum theory's challenge to causality and determinism that today look like a bit of an intellectual stretch.

Mermin is not alone in admitting such debates back into science. In a Perspective article on page 443, physicists Artur Ekert and Renato Renner place current work on quantum cryptography in a broader context that encompasses the thorny concept of free will.

Quantum methods of encoding information, they argue, combined with "an arbitrarily small amount of free will are sufficient to conceal whatever we like". To enable foolproof secrecy, "free will is our most

valuable asset". This, too, is the kind of claim that a few decades ago would have risked being dismissed, if not ridiculed, as idle coffee-room chat. It reveals researchers' new boldness for engaging with the meanings and corollaries of quantum physics. In doing so, they enrich the discussion as Bohr, Einstein and their colleagues did.

But why now? Interest in the founda-

"Researchers have a new boldness for engaging with the meanings and corollaries of quantum physics." tions of quantum theory - what it really tells us about the character of the world that we experience in reassuringly classical terms - has flourished since the late 1980s. That has been driven partly by the development of experimental techniques, especially in quantum optics, that can test ideas about phenomena such as entanglement (the codependence of remote quantum states), measurement and wavefunction collapse, and which were previously accessible only to theoretical speculation. As a result, physicists can more clearly see the most fundamental features of quantum theory - in particular the nonlocality and contextuality (contingency on how results are obtained) of quantum systems.

The other driver is an emphasis on quantum theory as a theory of information: of what we can know, transmit and share. This view has already thrown up practical applications such as quantum cryptography and rudimentary quantum computers. But it has also reawakened long-deferred foundational questions in new guises. It shows us that Bohr and Einstein could already see the ramifications for the philosophy and epistemology of science. Lacking the experimental tools to make progress, they doubted that these issues could ever be much more than metaphysical. Now they can be, and it is right that scientists should have the confidence to raise them afresh.

\section{Wheat lag}

\section{Growth in yields of the cereal must double if the Green Revolution is to be put back on track.}

$\mathrm{W}$ heat is widely considered to be the world's most important crop, and Norman Borlaug knew a thing or two about how to grow it. The US agronomist developed varieties that could better resist disease and gave higher yields. In doing so, he saved an estimated one billion people from starvation.

This week marks a century since Borlaug's birth, so what better time to consider why millions still go hungry, and to ponder how the next Green Revolution can be kick-started? At a meeting in Mexico this week, organized by the International Maize and Wheat Improvement Center 